\section{Commentary: unusual causes of pulmonary hypertension}

\author{
A J Peacock
}

Pulmonary hypertension is often enigmatic in presentation. This is because the symptoms (fatigue, dyspnoea, syncope) and the signs (a loud pulmonary second sound, with or without tricuspid regurgitation) are vague and common to many diseases. Furthermore, symptoms of pulmonary hypertension may well be overshadowed by the symptoms of accompanying diseases and, in addition, because there is no sphygmomanometer for the pulmonary circulation, making a diagnosis requires cardiac catheterisation.

The usual causes of pulmonary hypertension are chronic hypoxic lung disease, left ventricular dysfunction, pulmonary thromboembolism, left to right shunts and, rarely, the condition of primary pulmonary hypertension which develops in young women and is essentially a diagnosis of exclusion.

To reach a diagnosis the clinician must be aware of the possibility. The clues include excessive shortness of breath with a relatively normal heart and lung examination; a fall in gas transfer factor on respiratory function testing which is greater than expected for any abnormalities of lung volumes or spirometric values; right axis on electrocardiography; enlarged pulmonary arteries on chest radiography; and, perhaps most usefully, the presence of tricuspid reflux and right ventricular dilatation on echocardiography. The tricuspid reflux can also be used as a non-invasive estimate of pulmonary artery systolic pressure. Once pulmonary hypertension is identified, then a cause must be found.

Investigations include a ventilation-perfusion scan to exclude pulmonary thromboembolism and pulmonary angiography if there is doubt; pulmonary function tests and arterial blood gas analysis, with or without computed tomographic scanning to exclude chronic lung disease; and echocardiography to assess left ventricular function and intracardiac shunt. Sometimes, despite these investigations, the diagnosis is not clear and other clues must be sought.

These diagnostic difficulties are exemplified by the two case reports reported in this issue. The first by Riemer et $a l^{1}$ describes a 58 year old man with systemic symptoms of diarrhoea, weight loss, arthralgia, and breathlessness who was found to have small bilateral pleural effusions, a pericardial effusion, and malabsorption. Surprisingly, he was also found to have a pulmonary artery systolic pressure of more than $60 \mathrm{mmHg}$ on echo/Doppler scanning. Lung function showed only mild restriction but a marked reduction in gas transfer factor, blood gas tensions were normal, as was the ventilation-perfusion scan. The diagnosis was made when, because of the malabsorption, a duodenal biopsy specimen was taken which showed infection with Whipple's bacillus. Treatment with antibiotics resulted in clinical improvement and normalisation of the echocardiogram and gas transfer factor.

The second case, described by Hibbert and Braude, ${ }^{2}$ was a 71 year old woman with a known history of breast carcinoma who presented with cough and breathlessness. She was severely cyanosed with a $\mathrm{Po}_{2}$ of only $44 \mathrm{mmHg}$ $(5.86 \mathrm{kPa})$ but her lung fields were clear clinically, on chest radiography, and CT scanning. Electrocardiography suggested right atrial hypertension and echocardiography showed a pulmonary artery systolic pressure of more than $60 \mathrm{mmHg}$, confirmed by cardiac catheterisation. The left ventricle was normal. A ventilation-perfusion scan showed reduced perfusion in all zones. Pulmonary angiography was described as normal. In this patient no diagnosis was made until necroscopic examination when multiple tumour microemboli were found, consistent with a primary tumour of the breast.

These two cases demonstrate the difficulties of diagnosing pulmonary hypertension. They were both patients with severe breathlessness but relatively clear lung fields on clinical examination and relatively normal chest radiographs and, in one case, a normal CT scan. The clue to pulmonary hypertension came from the reduced gas transfer factor in one case and the abnormal echocardiogram in both cases. The cause, however, was more difficult.

In one case the diagnosis was not made until the necroscopic examination and in the other the diagnosis was made by a coincidental gastrointestinal biopsy. In both cases the diagnosis would have been made by open lung biopsy. With the advent of video-assisted thoracic surgery perhaps more patients who present with these symptoms and signs will receive open lung biopsy which should give the diagnosis of the cause of pulmonary hypertension. In these two cases the outcome may have been no different but, with the advent of different forms of vasodilator therapy and chemotherapy, a definitive diagnosis is essential if we are to be able to deal with these rare but difficult cases.

1 Riemer H, Hainz R, Stain Ch, Dekan G, Feldner-Busztin $\mathrm{M}$, Schenk $\mathrm{P}$, et al. Severe pulmonary hypertension reversed by antibiotics in a patient with Whipple's disease. Thora 1997;52:1014-5.

2 Hibbert M, Braude S. Tumour microembolism presenting as "primary pulmonary hypertension". Thorax 1997;52: 1016-7. 